\title{
Excited meson spectroscopy and scattering from lattice QCD
}

\author{
Christopher Thomas* (for the Hadron Spectrum Collaboration) \\ Trinity College Dublin \\ E-mail: thomascemaths.tcd.ie
}

\begin{abstract}
I report on recent progress in using lattice QCD to study excited mesons. I summarise results from a new calculation of excited charmonia where by using novel techniques we have extracted an extensive spectrum, including states with exotic quantum numbers and non-exotic hybrids, with high statistical precision. The interesting phenomenology suggested by the results will be highlighted. I will also discuss work on mapping out energy-dependent scattering phase shifts which has application to the study of resonances in both the charm and light-meson sectors.
\end{abstract}

Xth Quark Confinement and the Hadron Spectrum

8-12 October 2012

TUM Campus Garching, Munich, Germany

\footnotetext{
* Speaker.
} 


\section{Introduction}

In order to test QCD and understand the excitation spectrum of hadrons within QCD, it is essential to make first-principles calculations of the spectrum and to compare these against experimental data. Lattice QCD provides an ab-initio method for performing calculations in the non-perturbative regime - QCD is discretised on a finite four-dimensional hypercubic space-time lattice, a Euclidean (imaginary-time) space-time metric is used and then quantities can be computed in the path integral formulation by using importance-sampling Monte Carlo methods. In the last few years there has been significant progress in using lattice QCD to study excited hadrons and in these proceedings I will briefly summarise some recent results from the Hadron Spectrum Collaboration. I begin in Section 2 with excited charmonium spectroscopy and then in Section 3 discuss a calculation of the $\pi \pi$ isospin-2 phase shift. Full details are given in Refs. [1,2]. Similar calculations of excited charm-light $(D)$ and charm-strange $\left(D_{s}\right)$ mesons are discussed elsewhere in these proceedings [3].

\section{Excited charmonia}

The spectroscopy of charmonium mesons is an area of active interest, both experimentally and theoretically, driven by a wealth of high-quality data. Over the last decade experiments have found states expected but hitherto unobserved, made more accurate measurements of existing states and discovered a number of new resonances which do not fit into the pattern expected by models, for example the $X(3872)$ and $Y(4260)$. Suggested interpretations include conventional quark-antiquark states appearing at unexpected masses, hybrid mesons where the gluonic field is excited, or systems with additional quark-antiquark pairs such as molecular mesons or tetraquarks. However, to date there has been no observation in the charmonium region of a resonance with manifestly exotic $J^{P C}$ quantum numbers, i.e. a combination of $J$ (spin), $P$ (parity) and $C$ (charge-conjugation) quantum numbers that cannot arise from solely a quark-antiquark pair. Experimental interest continues with BESIII, experiments at the LHC and the planned PANDA experiment at GSI/FAIR in Germany.

The spectrum of excited charmonia from a lattice QCD calculation with dynamical strange and degenerate up and down quarks corresponding to $m_{\pi} \sim 400 \mathrm{MeV}$ is shown in Fig. 1. The lattice is anisotropic with a temporal lattice spacing, $a_{t}$, finer than the spatial lattice spacing, $a_{s} \approx 0.12 \mathrm{fm}$, and $\xi=a_{s} / a_{t} \approx 3.5$. The spatial volume is $24^{3}$ in lattice units corresponding to a spatial extent $L_{s} \approx 2.9 \mathrm{fm}$. Only connected contributions to the correlator are included and full details of the calculation are given in Ref. [1]. Also shown in the figure are experimental masses taken from summary tables of the PDG review [4]; the $X(3872)$ is not shown because its $J^{P C}\left(1^{++}\right.$or $\left.2^{-+}\right)$has not yet been determined experimentally.

As discussed in [1], we do not see any clear evidence for multi-hadron states in our extracted spectrum. The calculation used operators containing fermion bilinears; to study multi-hadron states we plan to enlarge the basis of operators to include those with a larger number of fermion fields. We will then be able to apply Lüscher's method and its extensions to compute phase shifts and so, at least in the case of elastic scattering, determine the mass and width of resonances, something I will return to in Section 3. We note that states above threshold can have large hadronic widths 


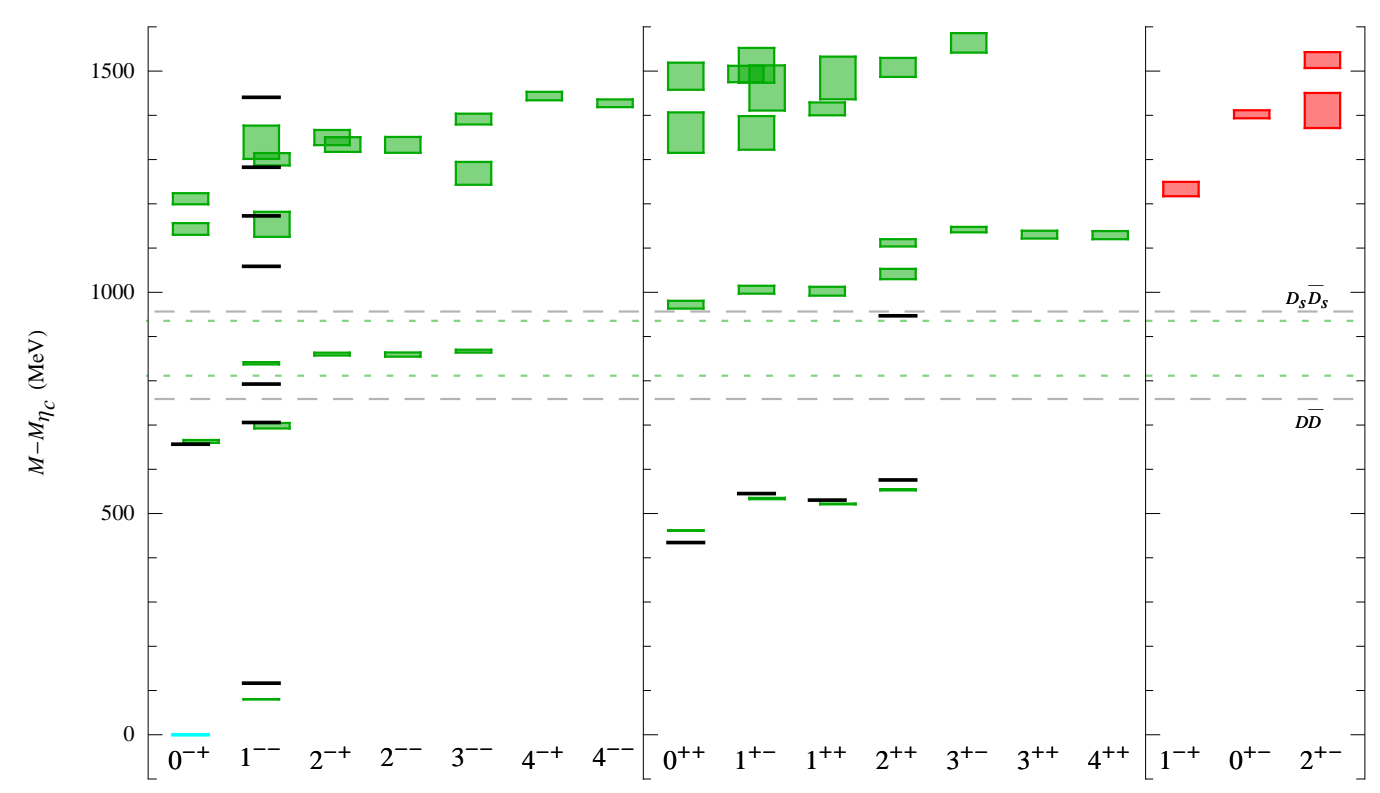

Figure 1: From Ref. [1]. Summary of the charmonium spectrum up to around $4.5 \mathrm{GeV}$ labelled by $J^{P C}$. The red and green boxes are the calculated masses and black lines are experimental values from the PDG summary tables [4]. The calculated (experimental) masses are shown with the calculated (experimental) $\eta_{c}$ mass subtracted. The vertical size of the boxes represents the one sigma statistical uncertainty on either side of the mean. The dashed lines indicate the lowest non-interacting $D \bar{D}$ and $D_{s} \bar{D}_{s}$ levels using calculated $D$ and $D_{s}$ masses (fine green dashing) and using the experimental masses (coarse grey dashing).

and a conservative approach is to only consider our current mass values accurate up to the hadronic width $[5,6]$.

The computed spectrum includes many states with exotic $J^{P C}$ quantum numbers and these are shown in the right panel of Fig. 1. Following Refs. [5, 7], by considering the overlaps of states onto interpolating operators, we identify these as hybrid mesons and also identify non-exotic hybrids. Fig. 2 shows the spectrum for only the $J^{P C}$ channels in which we identify candidates for hybrid mesons; the hybrid candidates are highlighted. In Ref. [1] we show that the pattern of hybrids can be interpreted as a colour-octet quark-antiquark pair coupled to a $1^{+-}$chromomagnetic gluonic excitation, with the lightest and first excited hybrid supermultiplets shown in Table 1. This is consistent with what was observed in the light meson sector [7]. We find that the lightest gluonic excitation appears at an energy scale $\sim 1.2-1.3 \mathrm{GeV}$ above the lightest conventional charmonium mesons. This is comparable to that found in the light meson [7] and baryon [8] sectors and suggests common physics.

\section{S- and D-wave isospin-2 $\pi \pi$ scattering phase shifts}

The vast majority of mesons can decay via the strong interaction to two or more lighter hadrons and therefore it is essential to understand resonances within QCD. This is relevant for most states in the light sector and for many new states in the charmonium region, e.g. the enigmatic $X(3872)$, 


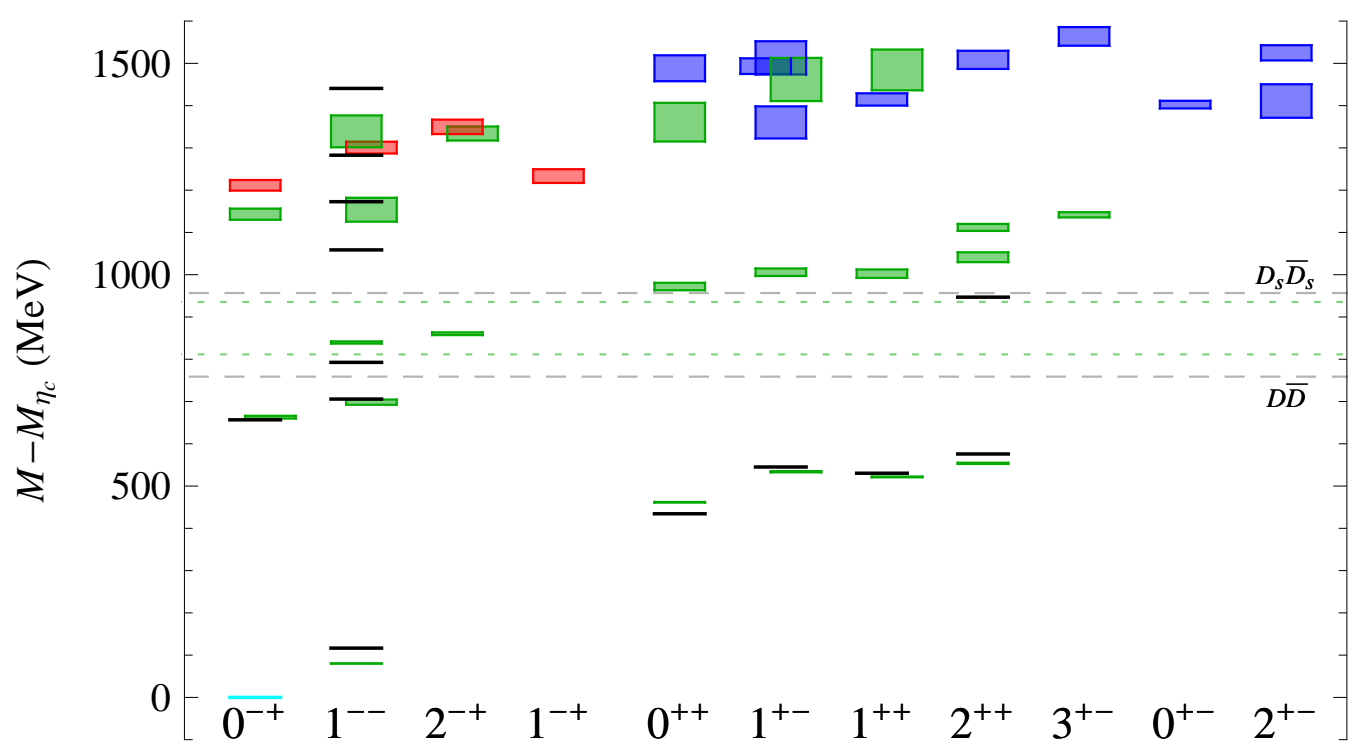

Figure 2: From Ref. [1]. Charmonium spectrum up to around $4.5 \mathrm{GeV}$ showing only $J^{P C}$ channels in which we identify candidates for hybrid mesons. Red (dark blue) boxes are states suggested to be members of the lightest (first excited) hybrid supermultiplet and green boxes are other states.

\begin{tabular}{cccc}
$J_{g}^{P_{g} C_{g}}$ & $L$ & $S$ & $J^{P C}$ \\
\hline \hline $1^{+-}$ & $0(S)$ & 0 & $1^{--}$ \\
& 1 & $(0, \mathbf{1}, 2)^{-+}$ \\
\hline \multirow{2}{*}{$1^{+-}$} & \multirow{2}{*}{$1(P)$} & 0 & $(0,1,2)^{++}$ \\
& & 1 & $(\mathbf{0}, 1,1,1, \mathbf{2}, \mathbf{2}, 3)^{+-}$ \\
\hline
\end{tabular}

Table 1: Hybrid supermultiplets for quark-antiquark pairs with spin $S$ and relative orbital angular momentum $L$, and where $J_{g} P_{g} C_{g}$ are the quantum numbers of the gluonic excitation; exotic $J^{P C}$ are shown in bold.

observed near or above decay thresholds. However, in the Euclidean formulation of lattice QCD direct access to dynamical properties is lost. The Lüscher method [9] and its extensions allow, in principle, at least in certain cases, indirect access to the infinite volume scattering parameters from the multi-hadron spectrum in a finite volume.

In Ref. [2] a combination of techniques was used to reliably determine the excited-state spectrum of two pions in a cubic box with high statistical precision. This allowed the Lüscher method to be applied to determine the $\pi \pi$ isospin-2 scattering phase shifts at a large number of kinematic points in the elastic region. For the first time in a lattice calculation, both $S$ and $D$-wave phase shifts were mapped out. The lattice ensembles were the same as those described above except that three spatial lattice volumes were used $\left(16^{3}, 20^{3}\right.$ and $24^{3}$ in lattice units). The extracted phase shifts are shown in Fig. 3 where $\delta_{\ell}$ is the phase shift in partial wave $\ell$.

The lattice data can be well described by a scattering length parameterisation, $p_{\mathrm{cm}}^{2 \ell+1} \cot \delta_{\ell}\left(p_{\mathrm{cm}}\right)=$ $\frac{1}{a_{\ell}}$, in both partial waves with $m_{\pi} a_{0}=-0.285 \pm 0.006$ and $m_{\pi}^{5} a_{2}=(-1.89 \pm 0.53) \times 10^{-6}$. Including also an effective range, $r_{0}$, in the S-wave phase shift, $p_{\mathrm{cm}} \cot \delta_{0}\left(p_{\mathrm{cm}}\right)=\frac{1}{a_{0}}+\frac{1}{2} r_{0} p_{\mathrm{cm}}^{2}$, gives a slightly improved fit with $m_{\pi} a_{0}=-0.307 \pm 0.013$ and $m_{\pi} r_{0}=-0.26 \pm 0.13$ but there is a high 


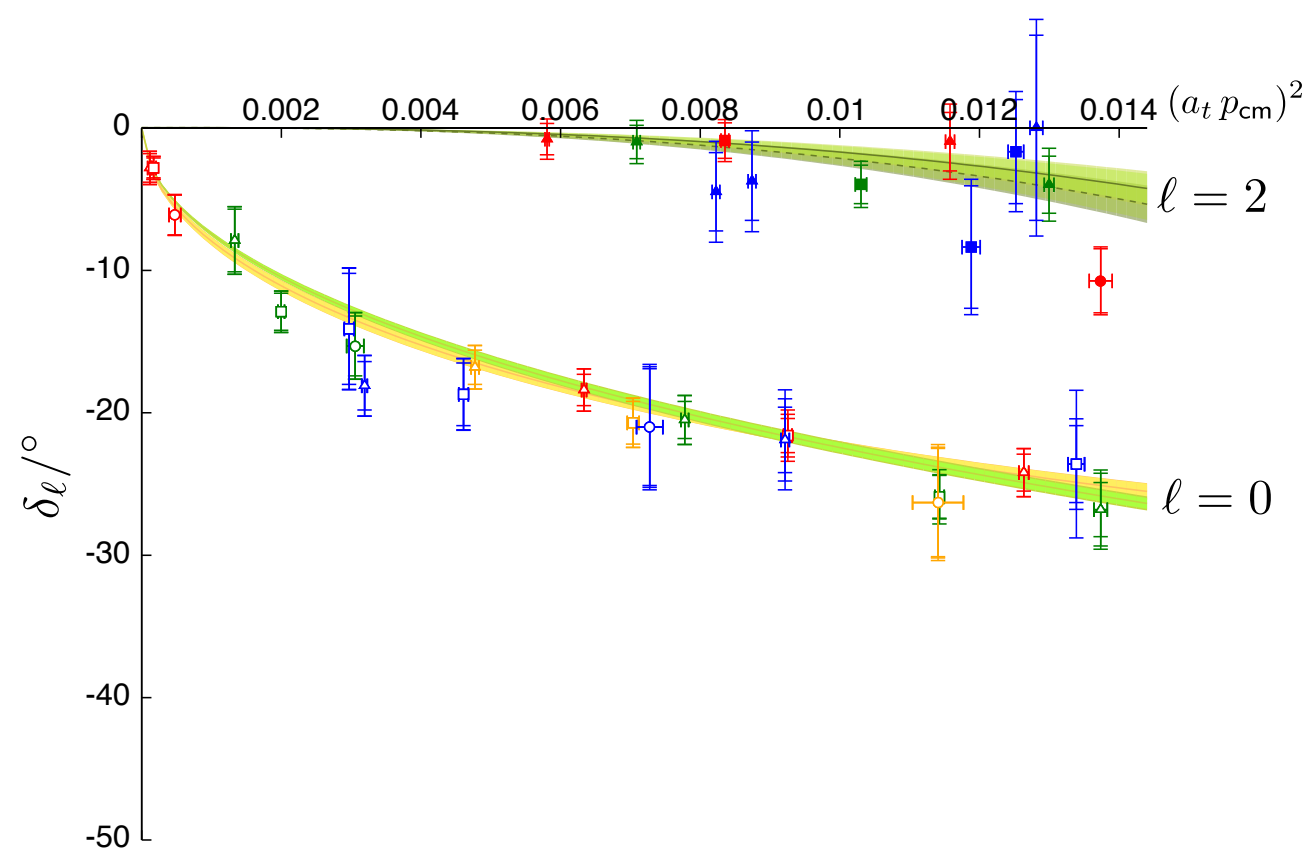

Figure 3: From Ref. [2]. Extracted isospin-2 $\pi \pi$ elastic scattering phase shift in $S$ and $D$-wave as obtained from analysis of finite-volume spectra with $m_{\pi} \sim 400 \mathrm{MeV}$. Center-of-momentum frame scattering momentum, $p_{\mathrm{cm}}$, expressed in units of the temporal lattice spacing, $a_{t}$. The momentum region plotted is entirely elastic, with the $4 \pi$ threshold opening at $\left(a_{t} p_{\mathrm{cm}}\right)^{2}=0.014$. Coloured points correspond to an analysis treating each energy level independently. The innermost errorbar is the statistical uncertainty, the middle errorbar reflects combined statistical uncertainty and uncertainty in $\left(a_{t} m_{\pi}, \xi\right)$ and the outermost errorbar shows the total uncertainty including imperfect knowledge of $\delta_{2,4}$ (all errors added in quadrature). Curves indicate a global analysis of all energy levels describing the phase shift by a scattering length or an effective range parameterisation.

degree of correlation between $a_{0}$ and $r_{0}(0.9)$. Statistically significant signals for elastic scattering were not observed in the $\ell=4$ partial wave. The techniques developed in Ref. [2] are a necessary input to future investigations of resonances in hadron-hadron scattering.

\section{Conclusions}

I have briefly summarised the results of some recent lattice QCD calculations at a single pion mass of $\sim 400 \mathrm{MeV}$. Future calculations will use lighted pion masses and larger volumes. Since I gave this talk, we have applied the methodology to a channel exhibiting a resonance for the first time, elastic isospin-1 $\pi \pi$ scattering [6]. We were able to map out the energy-dependence of the $\rho$ resonance in unprecedented detail and determined the resonance parameters. Following the very successful tests, we now plan to apply these techniques to other channels exhibiting resonances in the light and charm sectors. 


\section{Acknowledgments}

I thank my colleagues in the Hadron Spectrum Collaboration. Chroma [10] and QUDA [11, 12] were used to perform this work on the Lonsdale cluster maintained by the Trinity Centre for High Performance Computing funded through grants from Science Foundation Ireland (SFI), at the SFI/HEA Irish Centre for High-End Computing (ICHEC), and at Jefferson Laboratory under the USQCD Initiative and the LQCD ARRA project. Gauge configurations were generated using resources awarded from the U.S. Department of Energy INCITE program at the Oak Ridge Leadership Computing Facility at Oak Ridge National Laboratory, the NSF Teragrid at the Texas Advanced Computer Center and the Pittsburgh Supercomputer Center, as well as at Jefferson Lab. I acknowledge support from a Marie Curie International Incoming Fellowship, PIIF-GA-2010273320, within the 7th European Community Framework Programme.

\section{References}

[1] L. Liu et. al. (for the Hadron Spectrum Collaboration), Excited and exotic charmonium spectroscopy from lattice QCD, JHEP 1207 (2012) 126, [arXiv:1204.5425].

[2] J. J. Dudek, R. G. Edwards, and C. E. Thomas, S and D-wave phase shifts in isospin-2 pi pi scattering from lattice QCD, Phys. Rev. D86 (2012) 034031, [arXiv: 1203.6041 ].

[3] G. Moir et. al. (Hadron Spectrum Collaboration), Excited D and Ds meson spectroscopy from lattice $Q C D, P o S$ Confinement X (2012) 139.

[4] K. Nakamura et. al. (Particle Data Group), Review of particle physics, J.Phys.G G37 (2010) 075021. and 2011 partial update for the 2012 edition.

[5] J. J. Dudek, R. G. Edwards, M. J. Peardon, D. G. Richards, and C. E. Thomas, Toward the excited meson spectrum of dynamical QCD, Phys. Rev. D82 (2010) 034508, [arXiv: 1004.4930 ].

[6] J. J. Dudek, R. G. Edwards, and C. E. Thomas, Energy dependence of the $\rho$ resonance in $\pi \pi$ elastic scattering from lattice QCD, arXiv: 1212.0830.

[7] J. J. Dudek, The lightest hybrid meson supermultiplet in QCD, Phys. Rev. D84 (2011) 074023 , [arXiv:1106.5515].

[8] J. J. Dudek and R. G. Edwards, Hybrid Baryons in QCD, Phys.Rev. D85 (2012) 054016, [arXiv:1201.2349].

[9] M. Luscher, Signatures of unstable particles in finite volume, Nucl. Phys. B364 (1991) 237-254.

[10] R. G. Edwards and B. Joo (SciDAC Collaboration), The chroma software system for lattice qcd, Nucl. Phys. B. Proc. Suppl. 140 (2005) 832, [hep-lat/ 0409003 ].

[11] M. A. Clark, R. Babich, K. Barros, R. C. Brower, and C. Rebbi, Solving Lattice QCD systems of equations using mixed precision solvers on GPUs, Comput. Phys. Commun. 181 (2010) 1517-1528, [arXiv:0911.3191].

[12] R. Babich, M. A. Clark, and B. Joo, Parallelizing the QUDA Library for Multi-GPU Calculations in Lattice Quantum Chromodynamics, in International Conference for High Performance Computing, Networking, Storage and Analysis (SC), pp. 1-11, 2010. arXiv:1011.0024. 